\title{
BMJ Open New alumni EXperiences of Training and independent Unsupervised Practice (NEXT-UP): protocol for a cross- sectional study of early career general practitioners
}

\author{
Parker Magin, ${ }^{\oplus 1,2}$ Dominica Moad, ${ }^{\odot 1,2}$ Amanda Tapley, ${ }^{1,2}$ L Holliday, ${ }^{3}$ \\ Andrew Davey, ${ }^{1,2}$ Neil Spike, ${ }^{4,5}$ Kristen FitzGerald, ${ }^{6}$ Catherine Kirby, ${ }^{4,7}$ \\ Michael Bentley, ${ }^{6}$ Allison Turnock, ${ }^{8,9}$ Mieke L van Driel, ${ }^{\oplus}{ }^{10}$ Alison Fielding ${ }^{\oplus 1,2}$
}

To cite: Magin P, Moad D, Tapley A, et al. New alumni EXperiences of Training and independent Unsupervised Practice (NEXT-UP): protocol for a cross-sectional study of early career general practitioners. BMJ Open 2019;9:e029585. doi:10.1136/ bmjopen-2019-029585

- Prepublication history and additional material for this paper are available online. To view, please visit the journal (http:// dx.doi.org/10.1136/bmjopen2019-029585).

Received 3 February 2019

Revised 4 April 2019

Accepted 10 May 2019
D) Check for updates

(c) Author(s) (or their employer(s)) 2019. Re-use permitted under CC BY-NC. No commercial re-use. See rights and permissions. Published by BMJ.

For numbered affiliations see end of article.

Correspondence to Dr Parker Magin; parker.magin@newcastle. edu.au

\section{ABSTRACT}

Introduction General practice in Australia, as in many countries, faces challenges in the areas of workforce capacity and workforce distribution. General practice vocational training in Australia not only addresses the training of competent independent general practitioners (GPs) but also addresses these workforce issues. This study aims to establish the prevalence and associations of early career (within 2 years of completion of vocational training) GPs' practice characteristics; and also to establish their perceptions of utility of their training in preparing them for independent practice.

Methods and analysis This will be a cross-sectional questionnaire study. Participants will be former registrars ('alumni') of three regional training organisations (RTOs) who achieved general practice Fellowship (qualifying them for independent practice) between January 2016 and July 2018 inclusive. The questionnaire data will be linked to data collected as part of the participants' educational programme with the RTOs. Outcomes will include alumni rurality of practice; socioeconomic status of practice; retention within their RTO's geographic footprint; workload; provision of nursing home care, after-hours care and home visits; and involvement in general practice teaching and supervision. Associations of these outcomes will be established with logistic regression. The utility of RT0-provided training versus in-practice training in preparing the early career GP for unsupervised post-Ffellowship practice in particular aspects of practice will be assessed with $\chi^{2}$ tests.

Ethics and dissemination Ethics approval is by the University of Newcastle Human Research Ethics Committee, approval numbers $\mathrm{H}-2018-0333$ and $\mathrm{H}-2009-0323$. The findings of this study will be widely disseminated via conference presentations and publication in peer-reviewed journals, educational practice translational workshops and the GP Synergy Research subwebsite.

\section{INTRODUCTION}

Background and rationale

Access to best-practice primary care is the most important health systems determinant of the health of communities, and of healthcare
Strengths and limitations of this study

- The study will include early career general practitioners (GPS) recruited from three training organisations that educate and train $43 \%$ of Australia's GP vocational trainees.

- Though a cross-sectional study, questionnaire responses will be linked to previous in-training data reducing recall bias for training-related independent variables.

- Routinely collected in-training data have not been specifically collected for research purposes and this may result in incomplete data for some independent variables.

efficiency and equity. ${ }^{1}$ Access to, and quality of, primary healthcare in Australia is predicated on sufficient numbers and distribution of adequately educated and trained general practitioners (GPs). Shortages and maldistribution of the general practice workforce, however, are long-standing issues for primary healthcare delivery in many countries. ${ }^{1-4}$ It is a particular problem due to Australia's geography (with often significant distances between rural communities). The training period for GP registrars (trainees) provides an opportunity to influence the future work practices of GPs, and can assist in preparing GPs to respond to the medical, psychological and social needs within Australia's evolving primary healthcare system.

GP training in Australia is provided by regional training organisations (RTOs) and is overseen by the Royal Australian College of General Practitioners (RACGP) and the Australian College of Rural and Remote Medicine (ACRRM). RTOs are government-funded, not-for-profit GP training 
organisations. There are nine RTOs covering the whole of Australia. GP vocational training is 3 years (RACGP) or 4 years (ACRRM) in duration and includes hospital-based and general practice-based training. General practice-based training includes three 6 -month full-time equivalent compulsory general practice-based terms for RACGP registrars. Within each RTO, registrars train in accredited independent practices under the supervision of an experienced GP supervisor (trainer, preceptor). Registrars' educational programmes incorporate components of face-to-face one-on-one in-practice teaching sessions as well as structured away-from-practice teaching organised by their RTO.

In Australia, as in several other countries, rural and lower socioeconomic status (SES) communities have less access to GP services, contributing to poorer health outcomes. ${ }^{135-10}$ Government seeks to redress this access disparity within the general practice vocational training programme and workforce policies. The presumption is that experiencing training in rural and lower SES areas will encourage trainees to build an ongoing career in these areas. However, this relationship is complex, and research suggests a continuing trend towards urban practice-this is an ongoing issue in addressing workforce shortages. ${ }^{1112}$

Exacerbating workforce maldistribution, the evolution of general practice has seen an increase in demand for GP services. Within the Australian and international context, an ageing population, increasing chronic disease and multimorbidity prevalence have seen a rise in clinical complexity and increased clinical workloads. ${ }^{13-16}$ The provision of comprehensive care in this context includes increasing after-hours care (AHC), home visits (HV) and nursing home visits (NHV) for growing populations of frail aged, dementia and palliative care patients. ${ }^{14}$ 17-19 Despite this increase in community morbidity, retiring older GPs and a shift of all workers towards part-time, or less full-time hours, has meant GPs overall are working fewer hours. ${ }^{14021}$ Furthermore, a growing proportion of GPs are opting not to provide additional services, such as AHC and procedural care, despite financial incentives. $^{1417}$

Provision of vocational GP training has also been affected by changing work patterns. GP training is provided primarily through a workplace-based, apprenticeship-like model of learning which is predicated on an adequate number of senior GPs as supervisors. The role of teaching and learning within this apprenticeship model has become even more vital, and more demanding, by structural changes in the education and training experience of junior hospital doctors. ${ }^{22}$ There is, however, concern surrounding training capacity, with indications that there may not be sufficient senior GPs to take on such roles. ${ }^{23}$ Our previous research (the Readiness of Alumni for Practice Post Registrar Training-RAPPoRTproject), indicates that recent alumni (ex-registrars of GP training organisations) are engaging in teaching and training in the practice setting, and can be a vital part of the training of GP registrars. ${ }^{1724}$ Further investigation is warranted.

From a workforce planning perspective, it is critical to establish the prevalence of, and training programme predictors of, post-training outcomes. Greater understanding is also required of utility of training experience, and how training may inform, assist and influence subsequent independent practice. Ensuring registrars are exposed to a comprehensive patient-mix, and a wide scope of practice, assists in building confidence for future practice. The training period provides an opportunity to influence the future work practices of GPs, alumni continuing to work in general practice, uptake and retention in rural practice, and how they work, including AHC, HV, NHV, procedural and teaching and training workloads. To our knowledge, our previous RAPPoRT project ${ }^{1724}$ has been the only Australian study to date that has examined the location and nature of early career GPs' practice. International literature in this area is also limited. ${ }^{25}{ }^{26}$ We are not aware of any literature examining the influence of particular aspects of GP training on subsequent independent practice.

This study, New alumni EXperiences of Training and independent Unsupervised Practice (NEXT-UP): a cross-sectional study of early career general practitioners, will therefore build on our previous RAPPoRT study, to examine current practices of newly fellowed GPs, to help inform future GP training. Newly fellowed GPs are early career GPs who have recently graduated from their 'apprenticeship model' of vocational training where they work under the supervision of an experienced GP, before entering independent unsupervised practice.

The objectives of the project are to establish (1) characteristics of early career GPs' clinical practice, (2) associations of these characteristics, including associations with GP vocational training experiences and (3) early career GPs' perceptions of utility of training experiences for postfellowship practice.

\section{METHODS}

\section{Study design}

NEXT-UP will be a cross-sectional questionnaire-based study (see online supplementary appendix 1). Although being a cross-sectional study, data will be linked to data previously collected during the early career GPs' vocational general practice training.

\section{Study setting}

The study will include the former registrars ('alumni') of three RTOs-GP Synergy, Eastern Victoria GP Training (EVGPT) and General Practice Training Tasmania (GPTT).

Of these, GP Synergy is the largest, delivering education and training across the whole of the state of New South Wales and the Australian Capital Territory, with an intake of $\sim 500$ registrars per year. Of all Australian registrars in active general practice training, GP Synergy trains 
32.4\%, EVGPT (covering Eastern Victoria, including half of Melbourne) trains $7.7 \%$, and GPTT (covering the whole of Tasmania) trains $3.0 \% .{ }^{27}$ Within the three RTOs, the full range of Australian GP training settings are included, with practices located in all rural classifications. ${ }^{28}$ All three RTOs participate in the Registrar Clinical Encounters during Training (ReCEnT) project, ${ }^{29} 30$ which documents registrars' in-consultation clinical and educational experiences.

The RTOs deliver discrete educational sessions for the registrars, referred to hereafter as RTO-provided vocational training. This is concentrated in the registrars' first year where, on average, 1 day per month is spent out of practice at dedicated training sessions. The remainder of training takes place within an apprentice-like model in usually small, and often geographically dispersed, practices. We refer to this as in-practice vocational training. In-practice training is experiential, with registrars practicing as a GP but with recourse to seeking support and advice from their designated supervisor. The supervisor also delivers weekly 1 hour education sessions one-on-one with the registrar.

\section{Eligibility criteria}

Participants will be former GP Synergy, EVGP and GPTT alumni who achieved Fellowship between January 2016 and July 2018 inclusive. This represents registrars who had participated in the vocational training programme subsequent to the major restructure of GP training providers in Australia, which occurred in late 2015/ early 2016.

\section{Patient and public involvement}

Patients or members of the public were not involved as participants in this study.

\section{Outcomes}

Outcomes are:

1. The prevalence of early career GPs':

- Actively working in general practice.

- Practicing in rural and remote areas based on the Australian Standard Geographical ClassificationRemoteness $\mathrm{Area}^{31}$ of the practices location by postcode.

- Practicing in areas by SES based on Socio-economic Indexes for Areas-Index of Relative-Disadvantage

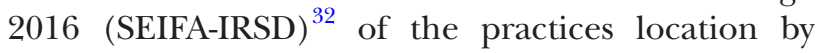
postcode.

- Retention within their RTO's geographic footprint (within the RTO's boundaries).

- Sessions of GP work undertaken on an average week. One session was defined as equalling $\sim 3.5$ hours, for example, a morning session. This will also be used as a dichotomous outcome-full-time (nine or more sessions per week) versus part-time.

- Provision of nursing home care.

- Provision of AHC, defined as rostered work apart from either weekday or Saturday morning scheduled sessions.
- Provision of HV.

- Provision of registrar supervision and/or provision of undergraduate supervision in general practice.

- Provision of advanced procedural skills.

i. Anaesthetics, including general, neurolept, major regional, epidural and spinal anaesthetics.

ii. Obstetrics, including normal deliveries, procedural intervention and operative obstetrics.

iii. Surgery, including surgical procedures requiring more than a basic infiltration of local anaesthetics.

- Non-clinical GP work. This includes research, and non-practice-based education, such as medical educator for a GP registered vocational training organisation, academic teaching role or other.

- Formal continuing education, including any education and/or training postfellowship (including qualifications currently being undertaken with the intention to continue to completion).

2. Alumni perceptions of the utility of particular aspects of their vocational training programme in preparing them for postfellowship practice, including:

- Structural aspects (supervision, in-practice teaching).

- Content (communication skills, EBM skills and so on).

\section{Participant timeline}

This will be a cross-sectional study. Recruitment will extend from those achieving Fellowship in January 2016 to mid-July 2018. Data collection will occur from mid-October 2018 to April 2019.

\section{Sample size}

We anticipate that $\sim 1300$ registrars of GP Synergy, EVGPT and GPTT who obtained fellowship between January 2016 and July 2018 (inclusive) will be eligible for inclusion. Of these, the majority will have trained with GP Synergy (anticipated to be 947), followed by EVGPT (236) and GPTT (140). Of these, expected prior participation in the ReCEnT study is $\sim 486$ of all eligible participants.

Assuming a $50 \%$ response rate, 650 registrars will enable estimation of proportions to within \pm 0.04 with $95 \%$ CI. For example, if the estimated proportion of rurally working registrars is 0.35 , a $95 \%$ CI for the proportion will be approximately $(0.31,0.39)$.

Statistical analyses will be conducted to identify factors associated with outcomes. For example, from RAPPoRT, ${ }^{11}{ }^{17} \sim 27 \%$ of alumni work rurally. The sample will provide $80 \%$ power at 0.05 significance to detect relative risks (RRs) in the range of 1.25-1.55, depending on the prevalence of explanatory variables. Alternatively, with $\sim 50 \%$ of alumni teaching/supervising or working part-time, the sample will enable detection of RRs in the range of 1.2-1.35.

\section{Recruitment}

Alumni will be recruited by their individual former RTOs using RTO-held contact details plus information available via publicly available sources (Australian Health 
Practitioner Regulation Agency (AHPRA) website, ${ }^{33}$ Yellow Pages, White Pages and so on).

The initial mailout will be conducted in October 2018 to all alumni who are eligible for participation at that time point. Rolling mailout will continue to registrars until April 2019, for alumni who have achieved Fellowship 6 months prior.

The initial invitation to participate will be sent via email and mail, followed by email reminders to those who did not respond. Two reminder emails will be sent out 2 and 4 weeks following the first email. This recruitment methodology has been successfully used in the RAPPoRT study. ${ }^{11} 17$

Alumni identified as living internationally will receive an email questionnaire only, due to costs of international mailing.

A \$A25 gift card will be offered for each alumnus who completes the questionnaire, as compensation for their time in participating.

\section{Data collection methods}

The NEXT-UP questionnaire was developed as an iteration of the RAPPoRT questionnaire. It was initially refined in light of the experiences with analysis of RAPPORT data. There was then wide consultation among the project team, which included extensive experience in general practice, including expertise in rural practice, and GP education and training.

The questionnaire was piloted, with a current senior registrar and a recent non-Australian medical graduate, for comprehensibility and timing. In piloting, the questionnaire took $7 \mathrm{~min}$ to complete.

An information pack with a cover letter, invitation to participate, information statement and questionnaire will be both emailed and posted to each potential participant. The mail-out and email-out will be conducted by the alumnus' individual former RTO.

A reply paid envelope will be provided with each mailed information pack. Alumni wishing to participate in the study will have the option of completing the questionnaire either in hard copy or electronically via SurveyMonkey.

For those choosing to complete the hard copy questionnaire, the alumnus will mail the completed questionnaire to the research team at GP Synergy.

As well as data elicited in the questionnaire, alumni will be asked for consent to link their questionnaire data with data routinely collected by their RTO during the alumnus' training period as a registrar, and data from the alumnus' participation in the ReCEnT project. ${ }^{29} 30$

Not all GP Synergy alumni will have participated in the ReCEnT project.

Alumni who do not consent to linkage of routinely collected and ReCEnT data will be requested to complete additional questionnaire items which will elicit, when practicable, data comparable to that to be accessed as routinely collected or ReCEnT data.
As well as the outcomes documented in the Outcomes section, questionnaire items will elicit the independent variables detailed in table 1 .

\section{Data management}

Each alumnus invitee will be assigned a study unique identifier. Master lists of alumnus names and alumnus unique study identifiers will be held at each of the RTOs in password-protected files, separate to password-protected data files.

Alumni unique study identifiers will be linked with the alumnus' ReCEnT project ID in a separate master list at each of the three participating RTOs.

GP Synergy will provide EVGPT and GPTT with a list of participating registrars, by way of study unique identifiers, for the purpose of extraction of that alumnus' routinely collected RTO data. These data, when extracted from GPTT and EVGPT databases will be provided to the research team at GP Synergy in deidentified form, identified only by study unique identifiers.

SurveyMonkey data will be downloaded by each RTO, with deidentified data sent to GP Synergy for storage in the study database at GP Synergy.

Data from returned hard copy questionnaires will be mailed directly to the research team at GP Synergy, where they will manually enter them into the online database via SurveyMonkey.

Routine training data extracted from RTO administrative databases will be checked and cleaned as appropriate to promote accuracy, reliability and consistency across participating RTOs.

\section{Statistical methods}

For outcomes relevant to these questions and listed in the Outcomes section:

1. Categorical outcomes prevalence will be calculated as proportions with $95 \%$ CI.

2. For continuous outcomes means with SD and a $95 \%$ CI will be calculated.

Then:

1. Associations of categorical outcomes will be assessed by univariate and multivariable logistic regression.

Multivariable analyses will be conducted with outcome factors:

- Practicing in rural and remote areas (defined as inner regional, outer regional, remote, very remote Australian Standard Geographical Classification-Remoteness Area (ASGC-RA) location of practice).

- Practicing in areas of lower SES (lowest quartile based on SEIFA-IRSD of the practices location).

- Retention within their RTO's geographic footprint.

- Workload (full-time vs part-time).

- Provision of nursing home care.

- Provision of AHC.

- Provision of HV. 
Table 1 Independent variables

Variable

Age

Gender

Identify as Aboriginal or Torres Strait Islander

Rural, regional or urban schooling background prior to university enrolment

Relationship status*

Spousal employment status*

Dependent children*

Primary language spoken at home

Australian/international medical graduate

Medical postgraduate years completed in an Australian

hospital prior to commencing GP term $1^{*}$

Type of fellowship

Year of fellowship

Training-related variables

Part-time/full-time status during training

Vocational training pathway

Leave, other than annual leave, taken during training

Rural location during training

Socioeconomic status of training location

Total number of practices worked in during training

Number of patients seen in terms 1, 2 and $3 \dagger$

Attendance at workshops/education sessions $\dagger$

Exam performance-fail in any RACGP/ACRRM component

Extended skills term undertaken in general practice

Performance of home visits, nursing-home visits, after-

hours care during training*

Practice size

Duration of consultationsł

Frequency of supervisor consultedł

Frequency of learning goals generatedł

\section{Definition}

Age at time of questionnaire completion.

Data extracted from RTO-held sources, where consent was provided. Otherwise selfreported by participants as male, female or unspecified. ${ }^{34}$

Dichotomous variable, of no to both, or yes to either Aboriginal and/or Torres Strait Islander Participants to nominate either rural, regional or metropolitan/urban schooling background.

Dichotomous variable of yes or no to currently living with a spouse or partner.

Employment status defined by partner/spouse currently working full-time, currently working part-time, not in the labour force or currently seeking work.

Dichotomous variable of yes or no to having dependent children.

Outcome of English, or other language, with provision for participant to specify language.

Outcome of Australian medical graduate, inclusive of New Zealand medical graduates as per RACGP policy, ${ }^{35}$ or international medical graduate as reported by participant.

As defined by participant in number of years.

FRACGP, and/or, FACRRM, and/or, FARGP.

Year of first fellowship.

Part-time-average of $<31.5$ hours per week per training term, not including after-hours care.

Dichotomous variable (general/rural) defined as the pathway the participant did the majority $(>50 \%)$ of their training.

Defined as more than a total of 6 weeks leave, apart from annual leave, across training. Leave was also defined specifically as per then current AGPT programme leave policy category 1-legislative leave, that is, parental, sick or carers leave, and/or category 2additional leave, including additional or personal leave. ${ }^{36}$

Based on practice postcode. Australian Standard Geographical ClassificationRemoteness Area $^{31}$

Based on practice postcode. SEIFA-IRSD $2016 .^{32}$

Count of practices across all training terms inclusive of extended skills if in general practice.

Total number of patients seen during the three core GP training terms.

Hours attended during GPT1 and GPT2.

Dichotomous variable of passed all exams first time, or failure of any exam.

Dichotomous variable of yes or no to completing a non-compulsory fourth term in general practice training.

Dichotomous variable of yes or no to performing $\mathrm{HV}$ and/or $\mathrm{NHV}$ and/or $\mathrm{AHC}$, during training.

After hours is defined as rostered work apart from either routine weekday or Saturday morning scheduled sessions.

Two dichotomous variables:

Trained in a small practice yes/no and

Trained in a large practice yes/no

Defined as $>13$ FTE weeks at a small and $>13$ FTE weeks at a large practice.

A small practice is defined as employing $<5$ doctors, while a large practice is defined as employing $>12$ doctors.

Due to variations in data collected across the three RTOs where head count data are not available, full-time equivalent, FTE, will be used.

Mean number of minutes over the three core training terms.

Percentage of consults/problems the supervisor was called in for over the three core training terms.

Percentage of consults/problems learning goals were generated for over the three core training terms. 
*Data elicited only via study questionnaire.

†Only applies to registrars consenting to use of routine RTO data.

†Only applies to registrars with ReCEnT data.

ACRRM , Australian College of Rural and Remote Medicine; AHC, after-hours care; FACRRM, Fellow Australian College of Rural and Remote Medicine; FARGP, Fellowship in Advanced Rural General Practice; FRACGP, Fellow Royal Australian College of General Practitioners; FTE, Full Time Equivalent; GP, general practitioner; GPT1, General Practice Term 1; GPT2, General Practice Term 2; HV, home visits; NHV, nursing home visits; RACGP, Royal Australian College of General Practitioners; ReCEnT, Registrar Clinical Encounters during Training; RTO, regional training organisation; SEIFA-IRSD 2016, Socio-economic Indexes for Areas - Index of Relative-Disadvantage 2016.

- Provision of registrar supervision and/or provision of undergraduate supervision in general practice.

The rating of the utility of RTO-provided vocational training versus in-practice vocational training in preparing the early career GP for unsupervised postfellowship practice will be tested univariately with $\chi^{2}$ tests. This will include the areas of:

1. Clinical knowledge.

2. Consultation skills.

3. Minor procedural skills.

4. Practicing evidence-based medicine.

5. Teaching skills.

6. Self-directed learning.

7. Reflective practice.

8. Professional responsibilities.

9. Tolerating clinical uncertainty.

10. Child and adolescent health.

11. Aged care.

12. Chronic disease management.

13. Patients with multimorbidity.

14. Aboriginal and Torres Strait Islander health.

15. Mental health.

Any change in statistical methods from this protocol will be acknowledged in papers reporting the analysis.

\section{Ethics and dissemination}

\section{Consent or assent}

Completion of the questionnaire will be deemed implied consent.

Additional consent provisions for collection and use of participant data in ancillary studies: Participants will be asked in the questionnaire if they consent to linkage of their data to routinely collected RTO data and (if applicable) ReCEnT study data.

\section{Confidentiality}

To protect the privacy and confidentiality of alumni from each participating RTO, potential participants will be identified and contacted by their former RTOs. All data supplied by GPTT and EVGPT to the researchers at GP Synergy will be deidentified. Each RTO will source current contact details using contact information previously provided to the RTO by the former-trainee, and will be supplemented by searching for current practice contact details from publicly accessible sources (AHPRA website, Yellow Pages, White Pages and so on).
Data will be identified only by a unique study identifier. Master lists of registrar names, and unique identifiers for registrars will be held at each of the three RTOs in password-protected files. The data will be kept in separate password-protected files. All data from completed questionnaires will be held by the research team at GP Synergy.

The participants' RTO will be informed of the completion of questionnaires using the participants unique study identifier. ReCEnT study data are regularly supplied to the researchers at GP Synergy by GPTT and EVGPT in deidentified form using the study unique identifiers. Data and other project documentation are saved in password-protected folders.

All the data will be stored on the GP Synergy Microsoft Azure Cloud account where data are encrypted.

\section{Access to data}

Only investigators and study statisticians will have access to study data. Advice from the approving ethics committee precludes making the database publicly available. Participants in earlier rounds of the ReCEnT project have not provided explicit consent for their data to be made publicly available.

\section{Dissemination policy}

The findings of this study will be disseminated via conference presentations and publication in peer-reviewed journals. It is anticipated that findings will be presented at general practice and medical education conferences.

We will also disseminate findings and promote research translation by conducting 'Research into Practice' translation workshops for stakeholders in Australian vocational training (medical educators, supervisors and so on).

Findings from the study will be communicated to participants via communication directly from their RTO.

Extensive documentation of results and methodological aspects will be made available to all interested parties on the GP Synergy Research subwebsite: https://research. gpsynergy.com.au/.

There will be no restrictions on publication or presentation to disseminate study findings to healthcare professionals and the public.

Publications will be written by the authoring team without use of professional writers. 


\section{Data availability}

Data will not be available externally due to HREC policy, as some of that data does not have permission from participants to be made available.

\section{DISCUSSION}

The work patterns of GPs are influenced by many complex factors. Our previous work suggests that future practice can be strongly influenced by training experiences. ${ }^{11} 1724$ To achieve best outcomes for GPs as well as communities, greater understanding of how training influences practice is necessary. The career choices of early career GPs with recent Fellowship provides an optimal opportunity for such research.

In this study, we aim to address the gap in knowledge surrounding early career GPs' practice postfellowship. We will do this by seeking to capture participants' demographic and practice data as well as their attitudes on the effectiveness and utility of their respective vocational training. It is expected that the findings will have generalisability to Australian RTOs, and should also have external validity to other countries with apprenticeship style GP training programmes.

These findings will be of importance in GP workforce planning, in policy decisions about distribution of registrars during training, activity requirements during training, RTO education programme content, supervision models, and in planning and policy around the interface of vocational training and continuing medical education.

Participating RTOs will benefit from use of this evidence to make structural changes to their training programmes, policies and practices, as well as to the content and means of delivery of their educational programmes.

Educationally, the findings from this study will provide RTOs with evidence on effects and utility of their programmes beyond the metrics of achievement of Fellowship of the RACGP or ACRRM and training programme completion. They will provide insight into which aspects of training alumni perceive as most useful in their subsequent independent practice. This will inform educational programmes.

The findings regarding prevalence and associations of retention of alumni within the geographic footprint will be of special interest and utility to practices, Primary Health Networks, health workforce planners and rural communities which seek to promote and incentivise local workforce recruitment and retention. The findings should be generalisable to the wider Australian GP vocational training programme, and the specific findings will apply to RTOs responsible for training $43 \%$ of Australia's GP registrars.

\section{Limitations}

A limitation of this study is that only a minority of alumni will have participated in the ReCEnT study. Analyses employing variables only available for ReCEnT-participating alumni will be done as separate subgroup analyses.
The data entry and data storage processes for routinely collected RTO data have not been designed for research purposes. The data are held in multiple forms which will require extraction and data cleaning.

\section{Author affiliations}

${ }^{1}$ Discipline of General Practice, School of Medicine and Public Health, University of Newcastle, Callaghan, New South Wales, Australia

${ }^{2}$ NSW and ACT Research and Evaluation Unit, GP Synergy Ltd - Newcastle, Mayfield West, New South Wales, Australia

${ }^{3}$ School of Medicine and Public Health, Faculty of Health and Medicine, University of Newcastle, Callaghan, New South Wales, Australia

${ }^{4}$ Eastern Victoria General Practice Training, Churchill, Victoria, Australia

${ }^{5}$ Department of General Practice, University of Melbourne, Parkville, Victoria, Australia

${ }^{6}$ General Practice Training Tasmania, Hobart, Tasmania, Australia

${ }^{7}$ School of Rural Health, Monash University, Clayton, Victoria, Australia

${ }^{8}$ Department of Health, Hobart, Tasmania, Australia

${ }^{9}$ Centre for Rural Health, University of Tasmania, Hobart, Tasmania, Australia

${ }^{10}$ Primary Care Clinical Unit, Faculty of Medicine, University of Queensland,

Brisbane, Queensland, Australia

Contributors PM, DM and AF conceived the NEXT-UP study. PM, DM, ATa, LH, $A D, N S, K F, C K, M B, A T u, M L v D$ and AF substantially contributed to the design of the study reported in this protocol. PM, DM and AF drafted this paper. This paper includes intellectual content of all authors, and all authors have provided final approval of the current version for publication.

Funding This research project is supported by the Royal Australian College of General Practitioners with funding from the Australian Government under the Australian General Practice Training program, grant number ERG020. In-kind support will be provided by GP Synergy, Eastern Victoria General Practice Training and General Practice Training Tasmania. The ReCEnT cohort study data which will be used in the NEXT-UP study, was funded until 2015 by the participating educational organisations: General Practice Training Valley to Coast, the Victorian Metropolitan Alliance, General Practice Training Tasmania, Adelaide to Outback GP Training Program and Tropical Medical Training, all of which were funded by the Australian Government. From 2016, ReCEnT is funded by an Australian Department of Health commissioned research grant and supported by GP Synergy.

Competing interests PM, AF, DM, ATa and AD are employees of GP Synergy. NS and CK are employees of Eastern Victoria General Practice Training. KF and MB are employees of General Practice Training Tasmania.

Patient consent for publication Not required.

Ethics approval Ethics approval is from the University of Newcastle Human Research Ethics Committee (HREC), approval number H-2018-0333. The ReCEnT study has HREC approval (University of Newcastle, Approval H-2009-0323).

Provenance and peer review Not commissioned; externally peer reviewed.

Open access This is an open access article distributed in accordance with the Creative Commons Attribution Non Commercial (CC BY-NC 4.0) license, which permits others to distribute, remix, adapt, build upon this work non-commercially, and license their derivative works on different terms, provided the original work is properly cited, appropriate credit is given, any changes made indicated, and the use is non-commercial. See: http://creativecommons.org/licenses/by-nc/4.0/.

\section{REFERENCES}

1. Starfield B, Shi L, Macinko J. Contribution of primary care to health systems and health. Milbank Q 2005;83:457-502.

2. Wass VGS, Petty-Saphon K. By choice-not by chance: supporting medical students towards future careers in general practice. London: Health Education England and the Medical Schools Council. 2016 https://www.hee.nhs.uk/sites/default/files/documents/By\% 20choice\%20-\%20not\%20by\%20chance.pdf (accessed 25 Mar 2019).

3. Gordon J, Valenti L, Bayram C, et al. An analysis of general practice encounters by socioeconomic disadvantage. Aust Fam Physician 2016;45:702-5.

4. Achat HM, Thomas P, Close GR, et al. General health care service utilisation: where, when and by whom in a socioeconomically disadvantaged population. Aust J Prim Health 2010;16:132-40. 
5. Butler DC, Petterson S, Bazemore A, et al. Use of measures of socioeconomic deprivation in planning primary health care workforce and defining health care need in Australia. Aust $J$ Rural Health 2010;18:199-204.

6. Harris MF, Zwar NA, Walker CF, et al. Strategic approaches to the development of Australia's future primary care workforce. Med J Aust 2011;194:88.

7. Katterl R. Socioeconomic status and accessability to healthcare in Australia: Primary Health Care Research \& Information Service. 2011 http://citeseerx.ist.psu.edu/viewdoc/download?doi=10.1.1.885 $6471 \&$ rep $=$ rep1\&type $=$ pdf (accessed 22 Jun 2018).

8. Australian Institute of Health and Wellbeing. Australia's Health 2014. In: Australia's health series. Canberra: AlHW, 2014.

9. Norbury M, Mercer SW, Gillies J, et al. Time to care: tackling health inequalities through primary care. Fam Pract 2011:28:1-3.

10. Mercer SW, Watt GC. The inverse care law: clinical primary care encounters in deprived and affluent areas of Scotland. Ann Fam Med 2007;5:503-10.

11. Magin P, Tapley A, Catzikiris N, et al. Where do GP registrars work after they complete training? Associations of rural practice location and regional retention. RMA17. Melbourne, 2017.

12. Britt HMG, Henderson J. General practice activity in Australia 201516. General practice series no. 40. Sydney: Sydney University Press, 2016.

13. Britt H, Miller GC, Valenti L, et al. The changing face of Australian general practice across the decades. Aust Fam Physician 2016;45:628-31.

14. Broadway B, Kalb G, Li J, et al. Do Financial Incentives Influence GPs' Decisions to Do After-hours Work? A Discrete Choice Labour Supply Model. Health Econ 2017;26:e52-e66.

15. Owen K, Hopkins T, Shortland T, et al. GP retention in the UK: a worsening crisis. Findings from a cross-sectional survey. BMJ Open 2019;9:e026048-e48.

16. Marchand $\mathrm{C}$, Peckham S. Addressing the crisis of GP recruitment and retention: a systematic review. Br J Gen Pract 2017;67:e227-e237.

17. Magin P, Catzikiris N, Tapley A, et al. Home visits and nursing home visits by early-career GPs: a cross-sectional study. Fam Pract 2017;34:77-82.

18. Pereira GJ. Palliative care in the hinterlands: a description of existing services and doctors' attitudes. Aust J Rural Health 2005;13:343-7.

19. van Kempen JA, Robben SH, Zuidema SU, et al. Home visits for frail older people: a qualitative study on the needs and preferences of frail older people and their informal caregivers. Br J Gen Pract 2012;62:e554-60.

20. Schofield DJ, Page SL, Lyle DM, et al. Ageing of the baby boomer generation: how demographic change will impact on city and rural GP and nursing workforce. Rural Remote Health 2006;6:1-9.

21. Joyce CM, Wang WC, Cheng TC. Changes in doctors' working hours: a longitudinal analysis. Med Care Res Rev 2015;72:605-21.
22. Wearne SM, Magin PJ, Spike NA. Preparation for general practice vocational training: time for a rethink. Med J Aust 2018;209:52-4.

23. Thomson JS, Anderson KJ, Mara PR, et al. Supervision--growing and building a sustainable general practice supervisor system. Med $J$ Aust 2011;194:S101.

24. Catzikiris N, Tapley A, Morgan S, et al. Maintaining capacity for inpractice teaching and supervision of students and general practice trainees: a cross-sectional study of early career general practitioners. Aust Health Rev 2018;42:643-649.

25. Jaakkimainen RL, Schultz SE, Glazier $\mathrm{RH}$, et al. Tracking family medicine graduates. Where do they go, what services do they provide and whom do they see? BMC Fam Pract 2012;13:26-36.

26. Sabey A, Hardy $H$. Views of newly-qualified GPs about their training and preparedness: lessons for extended generalist training. $\mathrm{Br} J \mathrm{Gen}$ Pract 2015;65:e270-e277.

27. Taylor R, Radloff A, Edwards D, et al. Australian general practice training program national report on the 2017 national registrar survey, 2018.

28. Australian Bureau of Statistics. 2039.0 - information paper: an introduction to Socio-economic Indexes of Areas (SEIFA). 2006 http://www.abs.gov.au/ausstats/abs@.nsf/mf/2039.0/ (Accessed 16 May 18).

29. Magin P, Morgan S, Henderson K, et al. The Registrars' Clinical Encounters in Training (ReCEnT) project: Educational and research aspects of documenting general practice trainees' clinical experience. Aust Fam Physician 2015;9:681.

30. Morgan S, Magin PJ, Henderson KM, et al. Study protocol: the Registrar Clinical Encounters in Training (ReCEnT) study. BMC Fam Pract 2012;13:50.

31. Australian Bureau of Statistics. 1216.0 - Australian Standard Geographical Classification (ASGC) Belconnen: ACT, 2011.

32. Australian Bureau of Statistics. Technical Paper Socio-Economic Indexes For Areas (SEFIA) 2011: Belconnen ACT: Commonwealth of Australia, 2013

33. Australian Health Practitioner Regulation Agency. Australian health practitioner regulation agency. 2018 www.ahpra.gov.au2018 (Accessed 22 Jun 2018).

34. Australian Government. Australian government guidelines on the recognition of sex and gender. Canberra: Attorney-General's Department, 2013.

35. Royal Australian College of General Practitioners. Working as a general practitioner in Australia; A step-by-step guide for international medical graduates working towards RACGP Fellowship. East Melbourne: RACGP, 2017.

36. Australian General Practice Training. AGPT Program Leave Policy 2017. In: Department of Health. http://www.agpt.com.au/About-Us/ Policies/AGPT-Policies-in-effect-1-January-2017 (Accessed 22 Jun 2018). 